\title{
FCR Provisions by Multi-Terminal HVDC System
}

\author{
Kosei Shinoda, Gianni Bakhos, Juan Carlos Gonzalez-Torres, Jing Dai, and Abdelkrim Benchaib
}

\begin{abstract}
Frequency control is considered to be an important function of multi-terminal HVDC (MTDC) systems. Sharing frequency containment reserves (FCRs) among interconnected AC systems using flexible HVDC technology is particularly attractive in the European context. However, coordination of the contribution between systems with different sizes, quality requirements and dynamic characteristics requires harmonized rules and well-defined control strategies. If the controllers are not properly designed, they can lead to disproportional supports, noncompliance with the existing regulation framework, or even degradation of the frequency quality. This paper analyzes several frequency control solutions for MTDC systems in terms of the adequacy of the expected contribution to the frequency containment process. The performance analyses are then confirmed using load-frequency models coupled with a three-terminal DC system. It is revealed that certain control solutions result in insufficient performance with respect to the desired FCR provision. The proposed distributed solution for each pair of converters can be the most compliant with the existing framework while keeping the highest degrees of freedom.
\end{abstract}

Index Terms-Frequency containment reserves, frequency control, high-voltage direct current (HVDC) transmission, multiterminal DC grids.

\section{INTRODUCTION}

$\mathbf{I}$ $\mathrm{N}$ an $\mathrm{AC}$ power system, the frequency plays a vital role in the system stability. For reliable and secure operation of the system, the frequency shall remain all the time within a permissible range stipulated by the transmission system operator (TSO). However, the proliferation of the non-synchronous power electronics devices and ongoing displacement of traditional power plants led to the decrease of the inertia, an inherent ability of the system to maintain the frequency and counteract power disturbances [1]. Moreover, the intermittent nature of the renewable generations increases the uncertainty in the system operation and threatens the security of the overall system.

A proper frequency control is, therefore, one of the major challenges today. In the European context, the ancillary service for frequency control is hierarchically divided into frequency containment process, frequency restoration process, and reserve replacement.

In general, the activation of frequency containment reserves (FCRs), also customarily called primary frequency control, is the first control action to counteract a frequency excursion. Conventionally, the actions are mostly taken by the generation units. Upon detecting the frequency deviation caused by a power disturbance, the speed governor deployed in generator turbine deviates its power output in proportion to the frequency deviation. This control action is realized in a decentralized manner without the need of real-time communication. The participating units must guarantee the continuous availability of the allocated FCR and its full deployment in time when necessary. The requirements and activation thresholds depend on the TSO(s) of each synchronous area.

In addition to the main providers of FCRs today - generators, power electronics-interfaced energy storage devices, and demand response - contributions from other flexible transmission system assets, such as HVDC systems, may be of great interest. HVDC transmission systems, as admittedly the most viable solution for bulk power transmission over very long distances today, are increasingly being deployed around the world in response to the growing need for crossborder power transmission. Given the increasing number of the HVDC installations, it is also expected that those HVDC systems play important roles in ensuring secure power system operation [2].

Frequency support making use of the fast and flexible controllability of HVDC links has been, therefore, an intensive research subject [3], which includes frequency support by using offshore wind farms [4], emergency power control [5], and mutual cooperation between synchronous areas [6]. In Europe, the technical feasibility of inter-synchronous area cooperation for primary frequency control has been demonstrated by the pilot test using the BritNed HVDC interconnector, the first HVDC link between Great Britain and the Netherlands [7]. A substantial cost saving using HVDC lines for frequency support of Nordic system was reported in [8]. In [9], a trilateral cooperation between $\mathrm{CE}, \mathrm{GB}$ and Nordic synchronous areas for the frequency containment process through multiple HVDC links was analyzed. A possible extension of those works could be a further enhancement of cooperation with neighboring systems through multi-terminal HVDC (MTDC) grids, which have gained widespread attention as a preferred option to bring more reliability and flexibility to the power systems. The future deployment of MTDC grids in North Sea will likely serve a vital function in the security of neighboring systems [10]. Significant research has already been conducted in academia and demonstrates the effective reduction in the frequency deviation using MTDC grids [11], [12], [13].

Although the ancillary service for frequency control is well defined in [14] for the interconnected European power systems, its implementation and the method of procurement still differ among the countries. When it comes to cooperation between systems with different sizes, quality regulations, and dynamic characteristics, clear policy agreements among the involved stakeholders must be needed. Sharing FCR among asynchronous systems through MTDC grids requires harmonized rules and a common framework for market-based procurement process as well as well-defined control strategies. If the controllers are not properly designed, it may result in disproportional supports, noncompliance with the existing framework, or even degradation of the frequency quality.

In the CENELEC report [15], some important prerequisites 


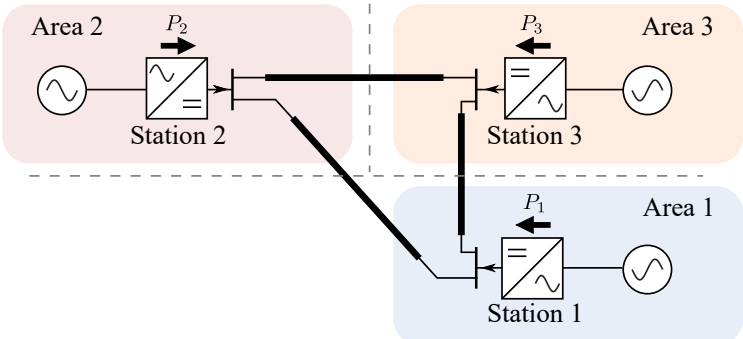

Fig. 1. Conceptual illustration of the studied hybrid AC/DC system.

for the FCR provisions using MTDC grids are discussed in a generic manner, (c.f. Chapter 4.4.4.2.2 of [15]). However, feasible control solutions and appropriate parameter selection to comply the contractual agreements are yet to be defined.

The paper analyzes the control strategy of the FCR provisions using MTDC grids in terms of compliance with the existing FCR framework. This paper first reviews several plausible control solutions and categorizes them in terms of implementation and pertaining communication needs. With the FCR to be the common basis for mutual frequency control coordination between systems with different regulatory rules, those solutions are analyzed through an analytical approach, in terms of how well the allocated FCR capacity is provided in case of a contingency. Finally, the analyses are confirmed by simulations of a trilateral FCR cooperation between three decoupled synchronous areas interconnected through an MTDC grid.

The rest of the paper is organized as follows. Section II gives the system model. Section III discusses the main parameters that characterize the FCR requirement. Section IV uses an analytical approach to study in depth 3 solutions of using MTDC system for frequency control coordination between asynchronous $\mathrm{AC}$ areas, where the exact expression of the effective frequency droop gain is derived, which shows the extent to which each solution is compliant with the expected behavior of FCR deployment. Section V gives simulations results and Section VI gives conclusions.

\section{SYSTEM MODEL}

We consider a hybrid AC/DC where $N_{c} \mathrm{AC}$ zones are connected exclusively to a DC grid by $N_{c}$ converters. An example of such a system with $3 \mathrm{AC}$ zones is shown in Fig. 1.

\section{A. AC system modeling}

In this paper, the aggregated load frequency model in [16] is considered. Its validity in capturing the general frequency dynamics has been demonstrated in [17] by comparison with actual measurements of the continental European system.

The time evolution of the frequency deviation $\Delta f_{i}$ in Zone $i$ is given by

$$
M_{i}^{a c} \frac{d \Delta f_{i}}{d t}=\Delta P_{i}^{g}-D_{i} \Delta f_{i}-\Delta P_{i}+P_{i}^{d i s}
$$

where $D_{i}$ in $\mathrm{W} / \mathrm{Hz}$ represents the self-regulating effect of the system load in Zone $i, \Delta P_{i}^{g}$ the aggregated power output

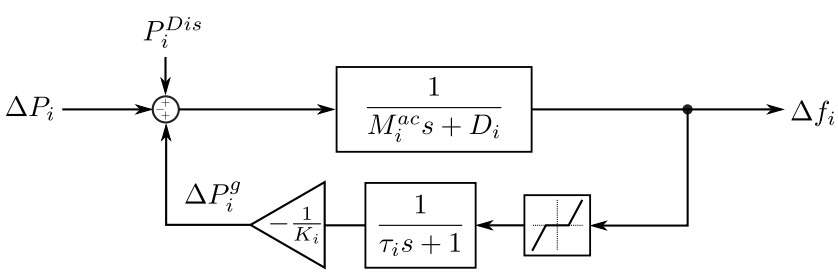

Fig. 2. Schematic depiction of of the synchronous area $i$.

variation of the participating generators of Zone $i, \Delta P_{i}$ the variation of the power injected from Zone $i$ to the DC grid by the relevant converter, $P_{i}^{d i s}$ the power disturbance which can come from the load side or the generation side, and $M_{i}^{a c}$ the inertia coefficient in $\mathrm{J} / \mathrm{Hz}$ defined as

$$
M_{i}^{a c}=\frac{2 H_{i}^{a c} S_{i}^{a c}}{f_{i}^{n o m}}
$$

with $H_{i}^{a c}$ the inertia constant in seconds, $S_{i}^{a c}$ the base power, and $f_{i}^{\text {nom }}$ the nominal frequency.

The aggregated contribution of the participating units to the primary frequency control is modeled according to the current network code for generator connection [18]. To be compliant with the grid code, the full-activation time of FCR, denoted by $\tau_{i}$, and the frequency insensitivity band $\left|\Delta f_{i}\right|$ are considered.

The model of the entire AC zone $i$ is depicted in Fig. 2, where $K_{i}$ is the droop constant of the aggregated generator.

\section{B. DC system modeling}

For analysis purpose, the losses in the DC system are commonly neglected, as in [19] and [20]. Then, dependency of the DC voltage deviation on the power variation of all the converters is given by

$$
M_{d c} \frac{d \Delta v_{d c}}{d t}=\sum_{i=1}^{N_{c}} \Delta P_{i}
$$

where $M_{d c}$ is the equivalent inertia of the system determined by the equivalent capacitance of the grid and the initial operating DC voltage.

The converter power variation $\Delta P_{i}$ has two components:

$$
\Delta P_{i}=\Delta P_{i}^{v}+\Delta P_{i}^{f}
$$

where $\Delta P_{i}^{f}$ is for the frequency control which will be studied in Section III and $\Delta P_{i}^{v}$ is for the DC voltage control in form of a droop:

$$
\Delta P_{i}^{v}=-G_{i}^{v} \Delta v_{d c}
$$

where $G_{i}^{v}$ is the DC voltage droop gain of Converter $i$.

\section{Entire system model}

Fig. 3 shows the schematic illustration of the hybrid system model developed from the discussions above.

\section{FCR SIZING}

The responsibilities of the adequate procurement of FCRs and the proper functioning lie upon the TSO of the controlled area. 


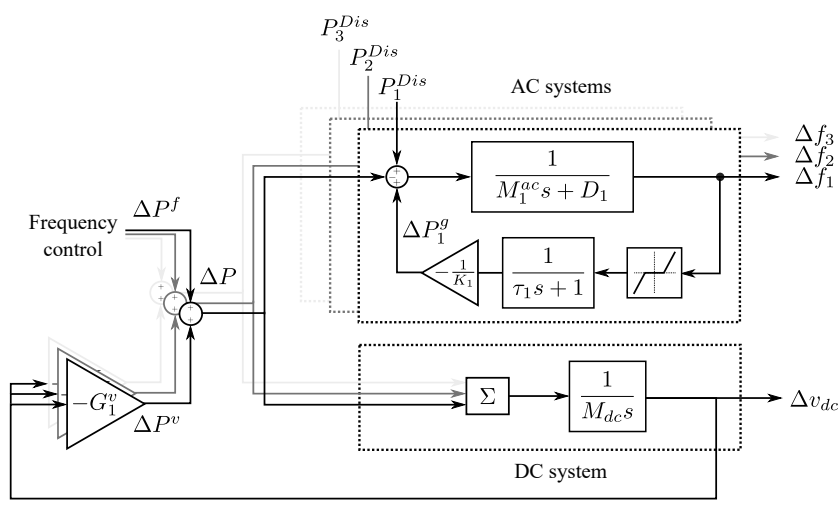

Fig. 3. Schematic depiction of the dynamic studied hybrid AC/DC system model.

\section{A. FCR parameters}

In [14], the main parameters that characterize the FCR requirement for ENTSO-E area are defined as follows:

- the reference incident

- the maximum steady-state frequency deviation

- the maximum instantaneous frequency deviation

- the FCR full activation time

The statutory frequency quality requirements as well as the activation speeds and thresholds vary from system to system. For instance, for the $\mathrm{CE}$ synchronous area, the reference incident is fixed at $3000 \mathrm{MW}$ in both positive and negative directions, whereas for GB and Nordic synchronous areas, the reference incident is defined as the largest imbalance that may result from a single incident in the system.

The maximum instantaneous frequency deviation is also an important quality factor. But whether the system can respect it or not greatly depends on the actual state of the system, especially the kinetic energy in the system at the moment of the incident [21]. In this paper, in order to discuss the generic requirement for MTDC systems, the focus is given to the steady-state deviation after frequency containment process instead of the transient extreme value.

\section{B. FCR procurement}

In order to contain the steady-state frequency deviation within the specified maximum steady-state frequency deviation, TSO of each area must procure an appropriate amount of FCR. The FCR is sized according to the reference incident without taking into account the self-regulation effects of loads [21]. Securing more FCR leads to a higher margin of security, but at the expense of higher procurement costs for operations.

In case of a frequency deviation equal to or larger than the maximum steady-state frequency deviation $\Delta f_{i}^{\max }$, the full capacity of the FCR must be effectively activated within a prescribed time (Article 154-7 in [14]).

As of 2021, roughly half of the FCR demand of CE grid is procured from the common auction platform. This was driven by the EU regulation [22] for consolidating the FCR procurement to increase liquidity while fostering effective competition, non-discrimination and transparency. Given the future participation of MTDC systems in the existing FCR cooperation framework, the MTDC systems must ensure the availability of the necessary transmission capacity during the operation and commit to the activation of the contracted FCR capacity in compliance with the established common and harmonized rules.

\section{Steady-State Analysis on Existing Solutions OF FREQUENCY CONTROL}

This section analyzes several control solutions for FCR provision by means of an analytical approach with the aim of understanding their essential features. The focus is given to the compliance with the expected behavior, in particular, in steady state after being subjected to a severe disturbance.

For simplicity, we consider the $3 \mathrm{AC}$ zones interconnected by a DC grid shown in Fig. 1. First, we derive the state-space model of the entire system. Then, three different frequency control solutions are discussed in terms of the adequacy of the expected contribution to the frequency containment process. More specifically, we analyze how well the actual behavior matches the expected behavior when a certain reserve capacity is assigned to the converter station and the control parameters are accordingly set.

\section{A. State-space representation}

For simplicity, the deadband and the time delay blocks in the primary frequency control in Fig. 2 are neglected when building the state-space model.

Define

$$
\begin{aligned}
\boldsymbol{x} & =\left[\Delta f_{1}, \Delta f_{2}, \Delta f_{3}, \Delta v_{d c}\right]^{T} \\
\boldsymbol{u} & =\left[\Delta P_{1}^{f}, \Delta P_{2}^{f}, \Delta P_{3}^{f}\right]^{T} \\
\boldsymbol{w} & =\left[P_{1}^{d i s}, P_{2}^{d i s}, P_{3}^{d i s}\right]^{T} .
\end{aligned}
$$

Then, from the equations in Section II, the state-space model can be found as

$$
\dot{\boldsymbol{x}}=\boldsymbol{A} \boldsymbol{x}+\boldsymbol{B} \boldsymbol{u}+\boldsymbol{E} \boldsymbol{w}
$$

where

$$
\begin{gathered}
\boldsymbol{A}=\boldsymbol{M}^{-1}\left[\begin{array}{ccc|c}
-\lambda_{1}^{a c} & 0 & 0 & G_{1}^{v} \\
0 & -\lambda_{2}^{a c} & 0 & G_{2}^{v} \\
0 & 0 & -\lambda_{3}^{a c} & G_{3}^{v} \\
\hline 0 & 0 & 0 & -\lambda_{d c}
\end{array}\right], \\
\boldsymbol{B}=\boldsymbol{M}^{-1}\left[\begin{array}{ccc}
-1 & 0 & 0 \\
0 & -1 & 0 \\
0 & 0 & -1 \\
\hline 1 & 1 & 1
\end{array}\right], \quad \boldsymbol{E}=\boldsymbol{M}^{-1}\left[\begin{array}{lll}
1 & 0 & 0 \\
0 & 1 & 0 \\
0 & 0 & 1 \\
0 & 0 & 0
\end{array}\right]
\end{gathered}
$$

with $\lambda_{i}^{a c}=\frac{1}{K_{i}}+D_{i}$ for $i=1,2,3, \lambda_{d c}=\sum_{i=1}^{3} G_{i}^{v}$, and

$$
\boldsymbol{M}=\operatorname{diag}\left(M_{1}^{a c}, M_{2}^{a c}, M_{3}^{a c}, M_{d c}\right) \text {. }
$$

Hereinafter, the steady-state values of the state variables are denoted by their corresponding symbols added by the superscript "ss".

Note that although the blocks of the dead-band and the time delay in the primary frequency control were neglected when building the above state-space model, the steady-state values of its state variables are the same as for the model when these two blocks are considered. 


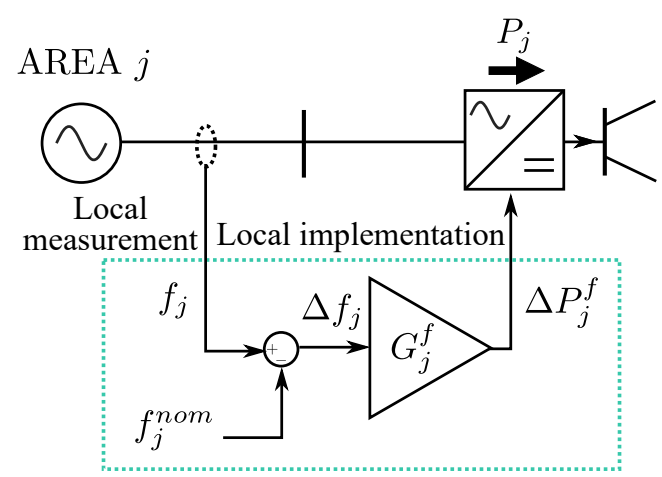

Fig. 4. The block diagram representation of Solution 1.

\section{B. Solution 1: Local control}

The most widespread solution in the literature consists of the frequency droop actions based on local measurement. We consider the case where the locally implemented frequency droop controller is combined with the DC voltage droop control [23], where no real-time communication is needed [24]. Since the AC frequency droop and DC voltage droop actions are in opposite direction, their interference creates a disruptive side-effect [25].

a) Control law: Among the numerous variants of frequency droop in the literature [26], we consider the following expression of the frequency droop control for Converter $j$

$$
\Delta P_{j}^{f}=G_{j}^{f} \Delta f_{j}=G_{j}^{f}\left(f_{j}-f_{j}^{n o m}\right)
$$

where $G_{j}^{f}$ is the frequency droop gain of Converter $j$, and $f_{j}^{n o m}$ is the nominal frequency. Fig. 4 gives its block diagram. With this solution, there are $N_{c}$ degrees of freedom corresponding to the droop gains of the $N_{c}$ converters.

b) Control gain design: When a certain FCR capacity, denoted by $\Delta P_{j}^{r e s}$, is assigned, it is expected to be fully activated when the maximum steady-state frequency deviation $\Delta f_{j}^{\max }$ is reached. Therefore, the requirement for the frequency droop gain can be expressed by

$$
G_{j}^{f}=\frac{\left|\Delta P_{j}^{\text {res }}\right|}{\left|\Delta f_{j}^{\text {max }}\right|} .
$$

c) Analysis: We consider the behavior of the system when one of the synchronous area is subjected to a severe disturbance.

Without loss of generality, suppose that only Zone 1 is disturbed by a disturbance, $P_{1}^{\text {dis }}$. By solving (7) and (8) for $\dot{x}=0$, the steady-state frequency deviation in Zone 1 after the disturbance can be expressed as

$$
P_{1}^{d i s}=\Delta P_{1}^{g}-D_{1} \Delta f_{1}^{s s}-\Delta P_{1}=\left(\lambda_{1}^{a c}+G_{1}^{f^{\prime}}\right) \Delta f_{1}^{s s}
$$

with

$$
G_{1}^{f^{\prime}}=G_{1}^{f}\left(1-\frac{G_{1}^{v}}{G_{1}^{v}+\sum_{i \neq 1} \frac{\lambda_{i}^{a c}}{G_{i}^{f}+\lambda_{i}^{a c}} G_{i}^{v}}\right) \leq G_{1}^{f} .
$$

Equation (11) shows that the effective frequency droop gain, $G_{1}^{f^{\prime}}$, is smaller than $G_{1}^{f}$, which means that the actual converter contribution is smaller than expected. In particular, when the frequency reaches the maximum allowable steadystate frequency deviation, i.e. $\left|\Delta f_{1}^{s s}\right|=\left|\Delta f_{1}^{\max }\right|$, we have

$$
\left|\Delta P_{1}\right|=\left|G_{1}^{f^{\prime}} \Delta f_{1}^{\max }\right| \leq\left|G_{1}^{f} \Delta f_{1}^{\max }\right|=\left|\Delta P_{1}^{\text {res }}\right| .
$$

In other words, the assigned FCR capacity is not fully exploited as expected.

The above analysis shows and it will be confirmed later in Section V that the main cause of this discrepancy is the interaction between the frequency droop and the DC voltage droop, whereby the frequency droop action of the disturbance zone results in an energy imbalance on the DC side, which is then shared among the stations equipped with a DC voltage droop controller, including the one for the disturbance zone.

\section{Solution 2: Local implementation with a common time- varying frequency reference}

To avoid the undesired interactions between the DC voltage control and the frequency control actions with Solution 1, at least some form of communication may be needed. While keeping the similar local frequency droop control structure in Fig. 4, the solution proposed in [27] uses a common time-varying frequency reference signal calculated from the global frequency measurements of all connected asynchronous AC systems. This reflects the overall frequency dynamics of all asynchronous AC systems while mitigating the undesired interactions with the DC voltage droop control.

a) Control law: This solution inherits the local frequency droop control structure

$$
\Delta P_{j}^{f}=G_{j}^{f}\left(f_{j}-f_{j}^{n o m} f_{p u}^{r e f}\right),
$$

where

$$
f_{p u}^{r e f}=\frac{\sum_{k=1}^{3} G_{k}^{f} f_{k}}{\sum_{k=1}^{3} G_{k}^{f} f_{k}^{n o m}} .
$$

With this solution, a converter observing a frequency lower than this common reference value will inject more power to the AC side, whereas it will withdraw more power from the $\mathrm{AC}$ side if the frequency is higher than the reference.

When all the AC zones have the same nominal frequency, as is commonly the case, this control law becomes

$$
\Delta P_{j}^{f}=\frac{1}{G_{\Sigma}^{f}} \sum_{k=1}^{3} G_{j}^{f} G_{k}^{f}\left(f_{j}-f_{k}\right)
$$

where $G_{\Sigma}^{f}=\sum_{k=1}^{3} G_{k}^{f}$. Interestingly, this means that the control action of one converter is the sum of the droop action on the frequency difference between this converter and each other one.

Fig. 5 illustrates the block diagram representation of this solution.

b) Control gain design: As before, with Solution 2, there are $N_{c}$ degrees of freedom corresponding to the frequency droop gains of the $N_{c}$ converters. Although some discussions on the parameter selection can be found in [27], no clear criterion for determining the parameters exists. Since this solution inherits the structure of the local frequency droop control, we consider that the minimum requirements on the reserve is the same as the previous solution given in (9). 


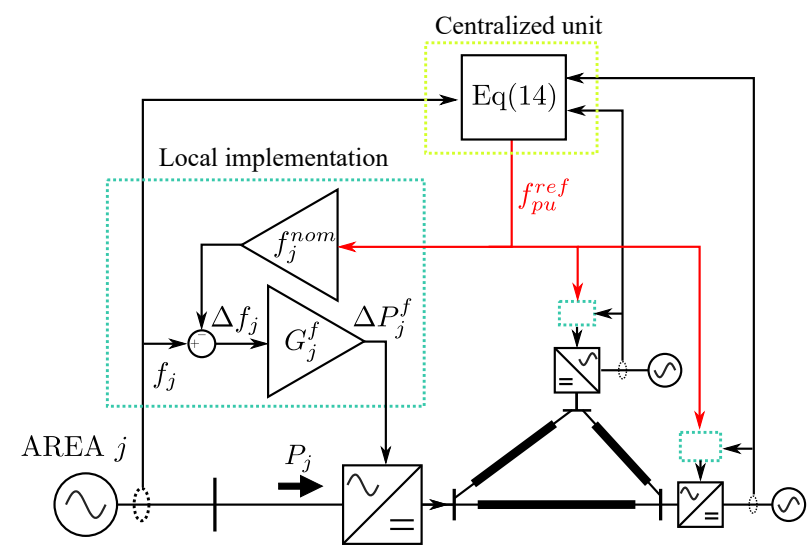

Fig. 5. The block diagram representation of Solution 2.

c) Analysis: By solving (7) with (15) for $P_{1}^{\text {dis }}$, the steady-state frequency deviation in Zone 1 is expressed by

$$
P_{1}^{d i s}=\left(\lambda_{1}^{a c}+G_{1}^{f^{\prime}}\right) \Delta f_{1}^{s s}
$$

with the effective frequency droop gain given in (17), which can be approximated as

$$
G_{1}^{f^{\prime}} \simeq G_{1}^{f}\left(1-\frac{G_{1}^{f}}{G_{\Sigma}^{f}}\right) .
$$

Unlike the previous solution, the effective frequency droop gain no longer depends on the DC voltage droop gains. This is because the communication through the dynamically calculated frequency reference allows to convey a frequency disturbance from one area to another without inducing undesirable DC voltage deviations. In other words, while the station connected to the disturbed AC system adjusts its energy exchange with the DC grid to support the frequency, the other stations compensate for the variation of the exchanged energy. Although it requires reliable communication between the centralized reference calculation unit and the converter stations, the above analysis proves that this solution can effectively decouple the frequency control from the DC voltage control.

On the other hand, it can be seen from (18) that the effective frequency droop gain is strongly influenced by its relative size of the assigned gain. In fact, (14) shows that as the relative size of the droop gain of an $\mathrm{AC}$ zone increases, the frequency of that zone becomes dominant in the weighted average frequency reference $f_{p u}^{r e f}$, which implies that the zone's frequency deviation with respect to $f_{p u}^{r e f}$ becomes less significant. The choice of the gains determines not only how much power a converter of the disturbed zone provides, but also how the other converters contribute to the compensation of the the energy imbalance. Therefore, in this solution, the gains must be carefully chosen taking into account both the required contribution to the local system and the contributions to the remote areas.

\section{Solution 3: distributed control}

The analysis on Solution 2 revealed that the mutual frequency support between AC grids through the weighted average frequency can be expressed as a superposition of the bilateral control action of each pair of AC grids. Based on this principle, another solution for frequency support is proposed here. The feature of the solution is a distributed controller for each pair of converters interfacing different synchronous systems. This solution is inspired from the primary frequency control scheme for HVDC line in [19] and the virtual link concept proposed for rotor-angle stability enhancement in [28].

a) Control law: The generic form of the proposed control law of the distributed solution for the pair of converters interfacing asynchronous grid $j$ and $k$ is given as

$$
\Delta P_{j k}^{f}=-\Delta P_{k j}^{f}=G_{j k}^{f} \Delta f_{j}-G_{k j}^{f} \Delta f_{k}
$$

and

$$
\Delta P_{j}^{f}=\sum_{\substack{k=1 \\ k \neq j}}^{3} \Delta P_{j k}^{f}
$$

Instead of relying on the local measurement only, the control law of this solution includes the frequencies measured at other converters. Each distributed controller contains two tunable gains, which allows to adjust the priority between the pairs while taking into account the differences in the frequency quality requirements of the different $\mathrm{AC}$ zones. When the distributed control modulates the power of one converter in a pair, the same amount of power is modulated at the other converter of the pair but in the opposite direction, hence no interference with the DC voltage. Fig. 6 shows the schematic illustration of this distributed controller.

b) Control gain design: In the proposed solution, the degrees of freedom in terms of control parameter is greatly increased to $N_{c}\left(N_{c}-1\right)$. On the one hand, such high degrees of freedom allow for unilateral frequency support of only one of the pair. For example, by choosing $G_{j k}^{f}>0$ but $G_{k j}^{f}=0$, the control only reacts to $\Delta f_{j}$ but not to $\Delta f_{k}$. On the other hand, in the case of multilateral frequency control coordination where the pair of asynchronous systems $j$ and $k$ agree on bilateral frequency containment control, a certain reserve capacity, denoted by $\Delta P_{j k}^{r e s}$, needs to be secured in the transfer capacity between the two systems. The reserved power is expected to be effectively activated in case of a disturbance

$$
G_{1}^{f^{\prime}}=G_{1}^{f}\left(1-\frac{G_{1}^{f}}{G_{\Sigma}^{f}}-\frac{G_{1}^{f} G_{2}^{f}\left(1+\frac{1}{G_{3}^{f}} \lambda_{3}^{a c}\right)+G_{1}^{f} G_{3}^{f}\left(1+\frac{1}{G_{2}^{f}} \lambda_{2}^{a c}\right)}{\left(G_{1}^{f}+G_{3}^{f}+\frac{G_{\Sigma}^{f}}{G_{2}^{f}} \lambda_{2}^{a c}\right)\left(G_{1}^{f}+G_{2}^{f}+\frac{G_{\Sigma}^{f}}{G_{3}^{f}} \lambda_{3}^{a c}\right)-G_{2}^{f} G_{3}^{f}}\right) \leq G_{1}^{f}
$$




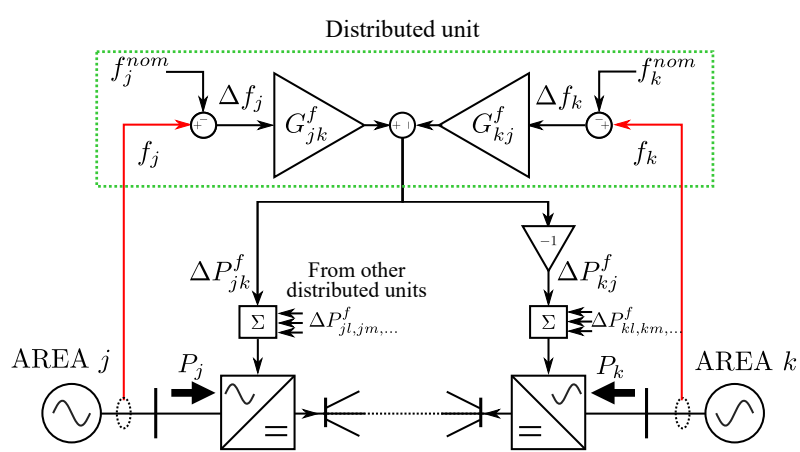

Fig. 6. The block diagram representation of Solution 3.

in either zone of the pair. The requirements on the frequency droop gains are thus,

$$
\begin{gathered}
G_{j k}^{f}=\frac{\left|\Delta P_{j k}^{\text {res }}\right|}{\left|\Delta f_{j}^{\text {max }}\right|}, \quad G_{k j}^{f}=\frac{\left|\Delta P_{j k}^{\text {res }}\right|}{\left|\Delta f_{k}^{\text {max }}\right|} \\
\left|\Delta P_{j}^{\text {res }}\right|=\sum_{\substack{k=1 \\
k \neq j}}^{N_{c}}\left|\Delta P_{j k}^{\text {res }}\right|
\end{gathered}
$$

c) Analysis: By solving (7) and (19) for $\dot{\boldsymbol{x}}=0$, the steady-state frequency deviation in Zone 1 after the disturbance can be expressed by

$$
P_{1}^{d i s}=\left(\lambda_{1}^{a c}+G_{12}^{f^{\prime}}+G_{13}^{f^{\prime}}\right) \Delta f_{1}^{s s}
$$

where the effective gains of the respective pair $G_{12}^{f^{\prime}}$ and $G_{13}^{f^{\prime}}$ are given in (23) and (24) respectively.

Similar to Solution 2, the effective frequency droop gain is no longer affected by the DC voltage droop parameters. Moreover, it can be seen that when the frequency droop gain of the converter is much smaller than the system powerfrequency characteristic, as it will likely be in reality, the difference between the effective droop gain and the actual value can be negligible. Another advantage of this solution is that each controller can be designed based on the bilateral contract between the pair of grids without interfering much with the other grids. In addition, the impact of the loss of communication between a pair is limited to that pair, thus increasing the overall reliability.

\begin{tabular}{|c|c|c|c|c|}
\hline \multirow{2}{*}{ Parameter } & \multicolumn{3}{|c|}{ Value } & \multirow[b]{2}{*}{ Unit } \\
\hline & $\begin{array}{l}\text { Area } 1 \\
\text { (CE) }\end{array}$ & $\begin{array}{c}\text { Area } 2 \\
\text { (Nordic) }\end{array}$ & $\begin{array}{l}\text { Area } 3 \\
\text { (GB) }\end{array}$ & \\
\hline Nominal frequency & 50 & 50 & 50 & $\mathrm{~Hz}$ \\
\hline Total system load & 380 & 37 & 25 & GW \\
\hline Inertia & 1930 & 369 & 378 & GWs \\
\hline Self regulating effect & 1.5 & 1.5 & 1.5 & $\%$ \\
\hline Aggregated droop & 0.3 & 0.2 & 0.18 & p.u./p.u. \\
\hline $\begin{array}{c}\text { Network power } \\
\text { frequency characteristic }\end{array}$ & 31033.3 & 4255.0 & 3152.8 & $\mathrm{MW} / \mathrm{Hz}$ \\
\hline Frequency insensitivity & 10 & 10 & 15 & $\mathrm{mHz}$ \\
\hline FCR full activation time & 30 & 30 & 10 & $\mathrm{~s}$ \\
\hline $\begin{array}{l}\text { FCR full activation } \\
\text { frequency deviation }\end{array}$ & \pm 200 & \pm 500 & \pm 500 & $\mathrm{mHz}$ \\
\hline
\end{tabular}

TABLE I

SYSTEM PARAMETERS ACCORDING TO [30][31]

\section{Demonstration by Simulations}

In this section, the discussions in the previous section on the three solutions are validated through simulations.

First, the load frequency model of three AC grids and the DC grid model are developed using the Modelica language. The parameters of the AC grids are given in Table I. The 640 $\mathrm{kV}$ MTDC system is modeled as an equivalent capacitor of $1 \mathrm{mF}$. The rating of each converter is assumed to be 1000 MW. The same DC voltage droop gain of 5000 MW/p.u. is assigned to each station such that the voltage deviation in case of a single converter trip will be contained within $10 \%$ [29].

The three solutions are implemented and compared in terms of the ability to properly activate a given reserve capacity. Here, we suppose that $\Delta P_{j}^{r e s}=400 \mathrm{MW}$ is contracted as the reserve capacity with each AC grid $(j=1,2,3)$. For Solution 3 , while maintaining consistency, the reserves are allocated to each pair of the grids, i.e. $\Delta P_{j k}^{r e s}=200 \mathrm{MW}$ for $j, k=$ $1,2,3$. Then gains are calculated according to (9) and (20) as summarized in Table II.

The studied scenario is a sudden 1000 MW loss of a generation in Area 2 at $t=20 \mathrm{~s}$.

In Fig. 7, the frequencies of three AC grids with different solutions are compared. As seen, the differences between the solutions are not significant. This is because any power deficit

$$
\begin{aligned}
& G_{12}^{f^{\prime}}=G_{12}^{f}\left\{1-\frac{G_{21}^{f}+G_{23}^{f} \frac{G_{31}^{f}}{\lambda_{3}^{a c}+G_{32}^{f}+G_{31}^{f}}}{\left\{\lambda_{2}^{a c}+G_{21}^{f}+G_{23}^{f}\left(1-\frac{G_{32}^{f}}{\lambda_{3}^{a c}+G_{32}^{f}+G_{31}^{f}}\right)\right\}}\right\} \leq G_{12}^{f} \\
& G_{13}^{f^{\prime}}=G_{13}^{f}\left\{1-\frac{G_{31}^{f}+G_{21}^{f} \frac{G_{32}^{f}}{\lambda_{2}^{a c}+G_{21}^{f}+G_{23}^{f}}}{\left\{\lambda_{3}^{a c}+G_{31}^{f}+G_{32}^{f}\left(1-\frac{G_{23}^{f}}{\lambda_{2}^{a c}+G_{21}^{f}+G_{23}^{f}}\right)\right\}}\right\} \leq G_{13}^{f}
\end{aligned}
$$


TABLE II

FCR PARAMETERS

\begin{tabular}{ccc}
\hline Solution $1 \& 2$ & \multicolumn{2}{l}{ Gain $(\mathrm{MW} / \mathrm{mHz})$} \\
\hline \hline Station 1 & $G_{1}^{F}$ & 2.0 \\
\hline Station 2 & $G_{2}^{F}$ & 0.8 \\
\hline Station 3 & $G_{3}^{F}$ & 0.8 \\
\hline
\end{tabular}

\begin{tabular}{ccccccc}
\hline Solution 3 & \multicolumn{3}{c}{ Gains of pair $(\mathrm{MW} / \mathrm{mHz})$} & \multicolumn{2}{c}{ Gain $(\mathrm{MW} / \mathrm{mHz})$} \\
\hline \hline Station 1 & $G_{12}^{F}$ & 1.0 & $G_{13}^{F}$ & 1.0 & $G_{1}^{F}$ & 2.0 \\
\hline Station 2 & $G_{23}^{F}$ & 0.4 & $G_{21}^{F}$ & 0.4 & $G_{2}^{F}$ & 0.8 \\
\hline Station 3 & $G_{31}^{F}$ & 0.4 & $G_{32}^{F}$ & 0.4 & $G_{3}^{F}$ & 0.8 \\
\hline
\end{tabular}

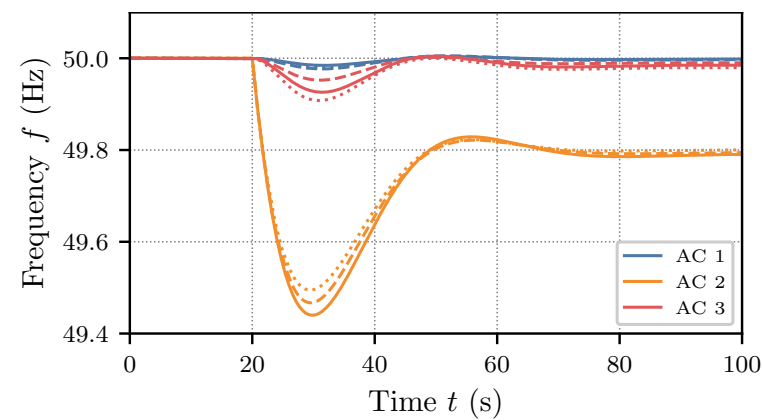

Fig. 7. AC frequencies: Solution 1 (solid), Solution 2 (dash), Solution 3 (dotted).

caused by non-compliance with the contracted performance will be compensated by the other FCR participating units (e.g. generators) with extra expense.

On the other hand, Fig. 8 shows the DC voltages during the event. It confirms that the autonomous frequency control actions in Solution 1 leads to DC side energy imbalance and the activation of DC voltage droop controls. In contrast, Solutions 2 and 3, which employ some communication, effectively avoid such undesirable DC voltage variations and the interference between the two droops.

In Fig. 9, 10, and 11, the active power contributions of the stations with each solution are depicted. The black dashed lines show the expected contractual contribution based on the allocated reserve of Station 2, which should be activated in proportion to the frequency deviation and the assigned gain.

In the case with Solution 1 shown in Fig. 9, there is a

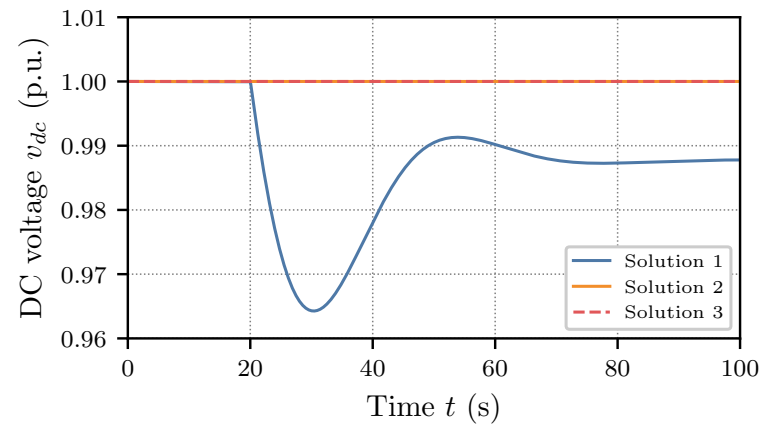

Fig. 8. DC voltages.

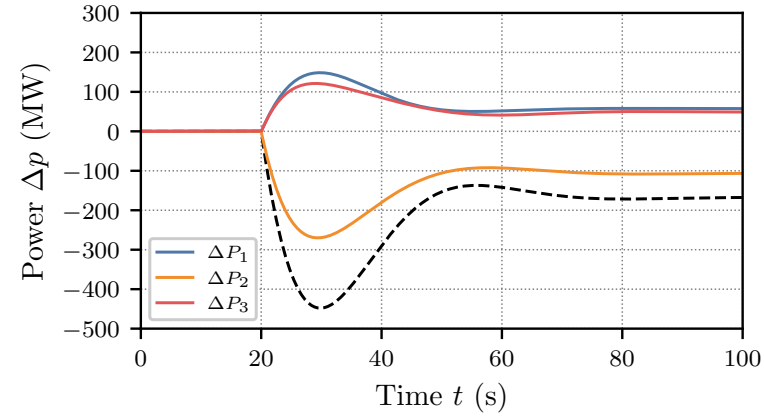

Fig. 9. Converter active power contribution - Solution 1.

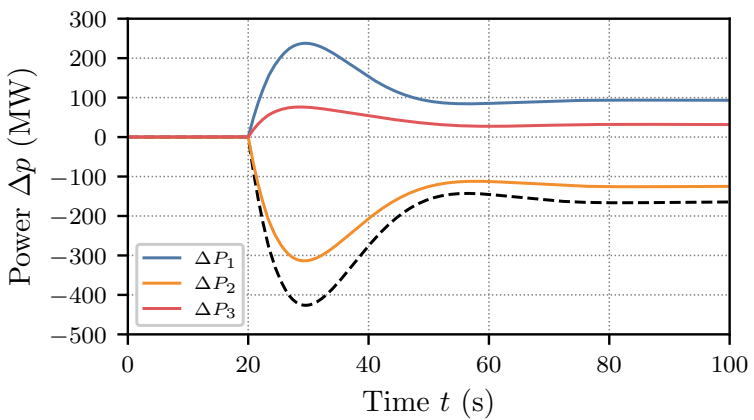

Fig. 10. Converter contribution - Solution 2.

noticeable difference between the expected contribution and the actual contribution delivered by Station 2. This is due to the interference with the DC voltage droop control. Depending on the assigned DC voltage droop gain, this deviation could be even larger.

Fig. 10 shows the result with Solution 2. Compared to the case with Solution 1, the insufficiency in the contribution is reduced but still recognizable. This confirms that, although the use of the weighted average frequency can effectively avoid the interaction with the DC voltage droop, it still cannot fulfill the expected activation of the assigned reserve with the conventional way of calculating the frequency droop gains. Therefore, the adequate droop calculation methods must be elaborated.

Fig. 11 shows the result with Solution 3. Compared to the previous cases, the adequacy of the contribution is visibly improved. Theoretically, the behavior of the controller implemented between a pair of grids is hardly affected by the gains of the controllers for the grids, which are not related to the pair.

Finally, Table III summaries the steady-state performance of the three solutions with respect to the expected contribution in proportion to the assigned droop gains and the frequency deviation. The estimated contribution of each solution derived using (11), (17), (23) and (24) coincides well with the simulation results, confirming the validity of the analyses.

\section{Conclusions}

In this article, the feasibility of frequency containment reserve provisions using an MTDC system was thoroughly investigated. Three different control strategies were analyzed in terms of their compliance with the requirement of the 


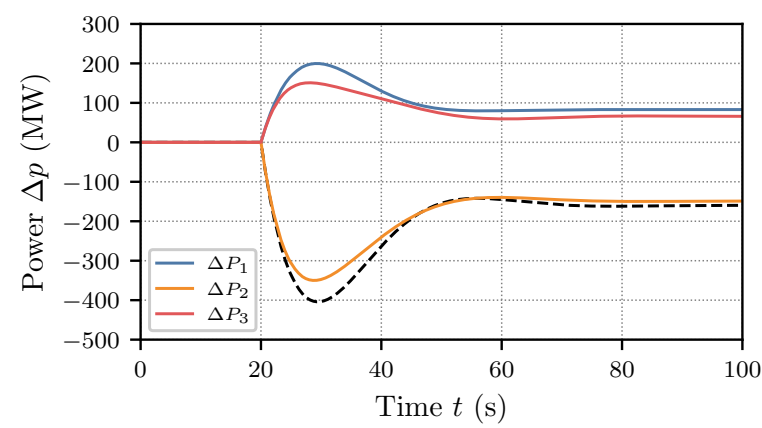

Fig. 11. Converter contribution: Solution 3.

TABLE III

COMPARISON OF STEADY-STATE PERFORMANCE OF STATION 2

\begin{tabular}{lcccc}
\hline & $\begin{array}{c}\text { Expected } \\
\text { contribution } \\
(\mathrm{MW})\end{array}$ & $\begin{array}{c}\text { Actual } \\
\text { contribution } \\
(\mathrm{MW})\end{array}$ & $\begin{array}{c}\text { Resulting } \\
\text { rate } \\
(\%)\end{array}$ & $\begin{array}{c}\text { Estimated } \\
\text { rate } \\
(\%)\end{array}$ \\
\hline \hline Solution 1 & -167.7 & -106.6 & 63.6 & 63.5 \\
\hline Solution 2 & -164.0 & -124.9 & 75.9 & 75.9 \\
\hline Solution 3 & -159.7 & -148.9 & 93.2 & 93.1 \\
\hline
\end{tabular}

full deployment of allocated FCR capacity for the maximum allowable frequency deviation. Each solution is studied analytically, where the implication of the frequency droop gain size is explained and the analytical expression of the effective frequency droop gain is provided. The simulation results showed that the distributed solution provides the most compliant FCR provision. As this control solution also offers robustness against partial loss of communication, it can be considered as a viable alternative to the conventional local frequency droop control.

\section{REFERENCES}

[1] P. Tielens and D. Van Hertem, "The relevance of inertia in power systems," Renew. Sustain. Energy Rev., vol. 55, pp. 999-1009, 2016.

[2] A. Kaushal and D. V. Hertem, "An overview of Ancillary Services and HVDC systems in European Context," Energies, vol. 12, no. 18, p. 3481, sep 2019.

[3] Y. Wen, C. Y. Chung, and X. Ye, "Enhancing Frequency Stability of Asynchronous Grids Interconnected with HVDC Links," IEEE Trans. Power Syst., vol. 33, no. 2, pp. 1800-1810, mar 2018.

[4] B. Silva, C. L. Moreira, L. Seca, Y. Phulpin, and J. A. P. Lopes, "Provision of inertial and primary frequency control services using offshore multiterminal HVDC networks," IEEE Trans. Sustain. Energy, vol. 3, no. 4, pp. 800-808, 2012.

[5] D. Obradovic, M. Dijokas, A. Tosatto, T. V. A. N. Cutsem, and R. Eriksson, "Assessment of HVDC Frequency Control Methods in the Nordic Test System," in CIGRE 2020, Paris, France, 2020, pp. 1-10.

[6] J. Machowski, P. Kacejko, Ł. Nogal, and M. Wancerz, "Power system stability enhancement by WAMS-based supplementary control of multiterminal HVDC networks," Control Eng. Pract., vol. 21, no. 5, pp. 583$592,2013$.

[7] S. P. Teeuwsen and R. Rössel, "Dynamic performance of the $1000 \mathrm{MW}$ BritNed HVDC interconnector project," IEEE PES Gen. Meet. PES 2010, pp. 1-8, 2010.

[8] A. Tosatto, M. Dijokas, T. Weckesser, S. Chatzivasileiadis, and R. Eriksson, "Sharing reserves through HVDC: Potential cost savings in the Nordic countries," IET Gener. Transm. Distrib., vol. 15, no. 3, pp. 480494, feb 2021.

[9] J. E. de Haan, C. Escudero Concha, M. Gibescu, J. van Putten, G. L. Doorman, and W. L. Kling, "Stabilising system frequency using HVDC between the Continental European, Nordic, and Great Britain systems," Sustain. Energy, Grids Networks, vol. 5, pp. 125-134, 2016.
[10] S. Chondrogiannis and M. P. Blanco, "Market Integration Scheme of a Multi-Terminal HVDC Grid in the North Seas," IEEE Trans. Power Syst., vol. 31, no. 3, pp. 2415-2422, 2016.

[11] N. R. Chaudhuri, R. Majumder, and B. Chaudhuri, "System frequency support through multi-terminal DC (MTDC) grids," IEEE Trans. Power Syst., vol. 28, no. 1, pp. 347-356, 2013.

[12] L. Papangelis, M. S. Debry, P. Panciatici, and T. Van Cutsem, "A receding horizon approach to incorporate frequency support into the AC/DC converters of a multi-terminal DC grid," Electr. Power Syst. Res., vol. 148, pp. 1-9, 2017.

[13] L. Papangelis, M. S. Debry, T. Prevost, P. Panciatici, and T. Van Cutsem, "Decentralized model predictive control of voltage source converters for AC frequency containment," Int. J. Electr. Power Energy Syst., vol. 98, pp. 342-349, 2018.

[14] COMMISSION REGULATION, "Establishing a guideline on electricity transmission system operation," 2017.

[15] CENELEC TC8X, "HVDC Grid Systems and connected Converter Stations. Guideline and Parameter Lists for Functional Specifications. Part 1," 2020.

[16] P. Kundur, Power System Stability and Control. McGraw-Hill, Inc., 1994.

[17] A. Díaz-García, C. Izquierdo, A. Cordón, F. Rodríguez, and R. Rivas, "Frequency stability model for energy transition studies in spain," in 2020 IEEE/PES Transmission and Distribution Conference and Exposition (T D), 2020, pp. 1-6.

[18] COMMISSION REGULATION, "Establishing a network code on requirements for grid connection of generators," p. 68, 2016.

[19] L. Harnefors, N. Johansson, and L. Zhang, "Impact on Interarea Modes of Fast HVDC Primary Frequency Control," IEEE Trans. Power Syst., vol. 32 , no. 2, pp. 1-1, 2016.

[20] S. G. Vennelaganti and N. R. Chaudhuri, "Ratio-based selective inertial and primary frequency support through MTDC grids with offshore wind farms," IEEE Trans. Power Syst., vol. 33, no. 6, pp. 7277-7287, 2018.

[21] D. Obradovic, M. Ghandhari, and R. Eriksson, "Assessment and Design of Frequency Containment Reserves with HVDC Interconnections," 2018 North Am. Power Symp. NAPS 2018, 2019.

[22] COMMISSION REGULATION, "Establishing a guideline on electricity balancing," 2017.

[23] T. M. Haileselassie and K. Uhlen, "Primary frequency control of remote grids connected by multi-terminal HVDC," IEEE PES Gen. Meet. PES 2010, pp. 1-6, 2010.

[24] R. Wiget, G. Andersson, M. Andreasson, D. V. Dimarogonas, and K. H. Johansson, "Dynamic simulation of a combined AC and MTDC grid with decentralized controllers to share primary frequency control reserves," in 2015 IEEE Eindhoven PowerTech. IEEE, jun 2015, pp. $1-6$.

[25] S. Akkari, M. Petit, J. Dai, and X. Guillaud, "Interaction between the Voltage-Droop and the Frequency-Droop Control for Multi-Terminal HVDC Systems," in 11th IET Int. Conf. AC DC Power Transm. Institution of Engineering and Technology, 2015, pp. 011 (7 .)-011 (7 .).

[26] M. Gu, L. Meegahapola, and K. L. Wong, "Coordinated Voltage and Frequency Control in Hybrid AC/MT-HVDC Power Grids for Stability Improvement," IEEE Trans. Power Syst., vol. 36, no. 1, pp. 635-647, 2021.

[27] Q. Zhang, J. D. McCalley, V. Ajjarapu, J. Renedo, M. Elizondo, A. Tbaileh, and N. Mohan, "Primary Frequency Support through North American Continental HVDC Interconnections with VSC-MTDC Systems," IEEE Trans. Power Syst., vol. 8950, no. c, pp. 1-1, 2020.

[28] J. C. Gonzalez-Torres, G. Damm, V. Costan, A. Benchaib, and F. Lamnabhi-Lagarrigue, "A Novel Distributed Supplementary Control of Multi-Terminal VSC-HVDC Grids for Rotor Angle Stability Enhancement of AC/DC Systems," IEEE Trans. Power Syst., vol. 36, no. 1, pp. 623-634, 2021.

[29] K. Shinoda, A. Benchaib, J. Dai, and X. Guillaud, "Over- and UnderVoltage Containment Reserves for Droop-Based Primary Voltage Control of MTDC Grids," IEEE Trans. Power Deliv., vol. 8977, no. c, pp. $1-1,2021$

[30] M. G. Ippolito, R. Musca, and G. Zizzo, "Analysis and Simulations of the Primary Frequency Control during a System Split in Continental Europe Power System,” Energies, vol. 14, no. 5, p. 1456, 2021.

[31] J. de Haan, "Cross-border balancing in Europe : ensuring frequency quality within the constraints of the interconnected transmission system," Ph.D. dissertation, Eindhoven University of Technology, 2016. 